\title{
Minimal pigment oculocutaneous albinism type 1
}

INSERM

\section{Source}

INSERM. (1999). Orphanet: an online rare disease and orphan drug data base. Minimal pigment oculocutaneous albinism type 1. ORPHA:352734

Type 1 minimal pigment oculocutaneous albinism (OCA1-MP) is an extremely rare form of OCA1 (see this term) with minimal pigment present, characterized by blond hair, variable iris transillumination, visual acuity rang ing from 20/80-20/200 and white skin, with or without skin nevi. 